\title{
Estudio de la formación de los precios de la tierra rural en los Municipios contiguos a Bogotá
}

\author{
Oscar Buitrago Bermúdez*
}

Resumen. En primera instancia se presenta la distribución espacial de los precios comerciales de la tierra rural en los municipios contiguos a Bogotá para el año 2000, como análisis previo a la determinación de los factores formadores del precio. Enseguida se presentan los resultados de los análisis multivariados realiza dos con un conjunto de variables que muy probablemente determinan el precio de la tierra en zonas rurales de influencia directa metropolitana, a partir de los cuales se determina la correlación entre ellas y con el precio catastral de los pre dios; dichos análisis se hicieron a partir de dos técnicas multivariadas: componen tes principales y regresión lineal múltiple. Se llega a concluir que la distribución espacial de los precios de la tierra en los municipios contiguos a Bogotá está defini da predominante por la red vial y un patrón de uso del suelo; y que los factores que determinan los precios de la tierra en la zona de estudio son la entidad terri torial político administrativa (es decir el municipio), el tipo de propietario y la accesibilidad, en contraposición a variables como el potencial agrícola, la presen cia de aguas o el relieve. Palabras clave: precios de la tierra, metropolización, geografía urbana, análisis multivariados.

* Ingeniero Agrícola, Universidad Nacional de Colombia. Magíster en Geografía - EPG. Convenio UPTCIGAC, Bogotá.

Perspectiva Geográfica 97 


\begin{abstract}
. at the first part, there appears the spatial distribution of the com mercial prices of rural land in municipalities contiguous to Bogota for the year 2000 , as analysis before of the determination of the forming factors of the price. Immediately there appear the results of the multivaried analyses realized with a set of variables that probably determine the price of the land in rural zones of direct metropolitan influence; this analyses let to establish the correlation among them and with the price of the land; the above mentioned analyses were done from two multivaried skills: principal components and linear multiple regression. There was concluded that the spatial distribution of land prices in the municipali ties contiguous to Bogota is defined predominantly by the net highways and the kind of use of the soil; and that the factors that determine the prices of the land in the zone of study are the municipality, the kind of owner and the accessibility, in contraposition to variables as agricultural potential, water presence or relief. Key words: prices of the land, metropolization, urban geography, multivaried analy ses.
\end{abstract}

Résume. Le étude de la distribution spatiale des prix commerciaux de la terre rural, dans les communes voisines a Bogotá a contribuée a l'identification des facteurs qui interviennent dans la formation des prix. Ensuite, on a selecctionnée des données qui probablement déterminent les prix des terres ruraux voisines aux grandes centres urbains.

D'autre part, pour le identification de la corrélation entre donneés et les prix de la terre, deux techniques d'analyses multivariés ont été utilisés: les composant principaux et la régression multiple lineaire. Les analyses ont donné comme résultat, que la distribution spatiale des prix de la terre des municipes voisines a Bogotá est définie par le réseau de voies et l'occupation du sol. Donc, le prix de la terre est fortement déterminé par facteurs comme, la commune où la propriété est localisée, le sorte de propriétaire et l'accessibilitée; neamoins, des caractéristiques comme, le potentiel agricole des sols, la présence d'eau et le type de relief où se trouve la propriétée, ne sont pas importants, même si, les terrains sont ruraux. Mots clés: prix de terre, accroissement urbaine, géographie urbaine, metropolization.

Aceptado: junio 2003.

98 Oscar Buitrago Bermúdez 


\section{Distribución espacial de los precios de la tierra en relación con la ocupación del suelo}

Para establecer la variación espacial de los precios comerciales de la tierra en el área de estudio y su relación con la ocupación del suelo, en primer lugar se concertaron entrevistas con agente inmobiliarios locales, quienes sobre mapas de su respectivo municipio delimitaron zonas homogéneas y asignaron precios promedios. La figura 1 es el resultado síntesis de la indagación en campo de los precios comerciales de la tierra en el área de estudio. Los anteriores resultados fueron confrontados con el mapa de usos obtenido por el CEDE (Centro de Estudios Económicos) y la Cámara de Comercio de Bogotá (1998).

En la figura 1 sobresale una estructura espacial de precios comerciales determinada longitudinalmente por tres ejes: la vía Cajicá - Chía - Cota - Funza y Mosquera, la vía Bogotá - La Calera Sopó, y la autopista Sur; y transversalmente, por la Autopista Medellín y la carretera de Occidente. Para efectos del análisis los ejes longitudinales se denominarán L1, L2 y L3, respectivamente, en tanto que los transversales de designarán como T1 y T2.

\section{Eje L1 Vía Cajicá - Chía - Cota - Mosquera}

Este eje presenta los mayores precios de toda el área de estudio y se encuentran en el municipio de Sopó y Chía sobre la autopista norte, cuyos datos oscilan entre $\$ 1.000$ y $\$ 2.500$ millones por hectárea, propiciados principalmente por la ocupación de vivienda suburbana de altos estratos, comercio especializado, clubes y áreas recreacionales.

En los municipios de Sopó, Chía y Cota, la presencia en las laderas de los cerros orientales de parcelaciones y condominios de muy baja densidad y alrededor de los clubes y zonas de servicios, activa la renta de segregación socioespacial, que se manifiestan en los elevados precios de estas tierras, con montos desde $\$ 250$ a $\$ 1.000$ millones por hectárea, muy por encima de lo que normalmente llegarían a tener en su condición de espacios rurales. En los condominios de las zonas quebradas, influye sobre el precio, la excelente perspectiva visual que desde allí se tiene sobre el paisaje de la Sabana.

En Chía, las zonas denominadas de granjas, cuyo uso en realidad está dominado por la vivienda suburbana, presentan densidades medias y precios unitarios considerablemente superiores a los encontrados en las montañas, desde $\$ 1.000$ a $\$ 1.500$ millones.

Sobresale el hecho de que los precios unitarios en el costado occidental de la Autopista y la vía Chía - Cajicá, alrededor de $\$ 2.000$ millones, sean más altos que en el costado oriental, cercanos a $\$ 1.500$ millones; 10 
anterior se explica por que las personas que salen de la ciudad prefieren abastecerse por el camino a su lugar de destino; este es un ejemplo de cómo se genera una renta diferencial por comercio, que al final es pagada por el consumidor de espacio para la actividad comercial.

En Cota, llama la atención el alto precio unitario de la tierra en los alrededores del casco urbano y especialmente en las cercanías al resguardo indígena, con valores que oscilan entre $\$ 400$ y $\$ 1.000$ millones, causado principalmente por el uso habitacional para estratos socioeconómicos cinco y seis. Estos precios también se encuentran sobre la vía que conduce a Siberia y sus alrededores, pero asociados a uso comercial y agroindustrial. Con menores precios sobresale, la vereda Vuelta Grande, como consecuencia de la combinación de actividades habitacionales para altos estratos, en la zona denominada Parcelas de Cota, y la a que ha sido designada en gran parte como zona industrial $y$ comercial.

En el segmento Siberia - Funza, aparece nuevamente una zona de elevados precios unitarios, alrededor de $\$ 2.000$ millones, ocasionada por la designación de un corredor industrial cuya función principal será el albergar empresas que por su actividad requieran cercanía al aeropuerto $\mathrm{El}$ Dorado. Esta zona industrial junto con la de Cota, forman un continuo que de ser fomentado, propiciará en un futuro la urbanización de esta área y por tanto, la pérdida definitiva de estas tierras aptas para la producción agropecuaria o para la preservación de cinturones amortiguadores del crecimiento de Bogotá.

En Funza se nota la influencia que sobre los precios unitarios de la tierra tiene el club de golf San Andrés, con valores cercanos a los $\$ 2.000$ millones.

En este eje, los menores precios comerciales de la tierra se presentan en el cerro de la Balvanera en Chía, considerado una reserva forestal, y en los territorios ocupados por el resguardo indígena que comparten Chía y Cota.

\section{Eje L2 Vía Bogotá - La Calera - Sopó}

Este eje se caracteriza por tener una intensidad de precios unitarios de la tierra inferior al anterior. Los mayores precios se encuentran asociados a conjuntos y parcelaciones de diferentes tamaños con densidades bajas, que se encuentran en el área de influencia de las vías, alrededor de el embalse de San Rafael y en el valle del río Teusacá.

El desarrollo de la vía Bogotá - La Calera por San Rafael, ha favorecido el aumento de los precios de la tierra en la vereda El Salitre contigua al límite político administrativo de Bogotá, encontrándose valores 
cercanos a los $\$ 350$ millones por hectárea.

El área de influencia que la carretera La Calera - Sopó, tiene sobre el valle del río Teusacá, determina un zona de precios relativamente homogéneos, cuyos valores oscilan entre $\$ 85$ y $\$ 150$ millones por hectárea, asociados principalmente a actividades agropecuarias, pero también ocurren algunas excepciones que se relacionan con la presencia de condominios y las áreas vecinas al casco urbano de Sopó, en donde los precios unitarios llegan a duplicarse.

Los menores precios unitarios de la tierra se presentan en las laderas de las montañas que forman el valle del río Teusacá, por ser áreas de páramos, subpáramos y nacimientos de aguas.

\section{Eje L3 Autopista Sur}

Este eje de precios de la tierra está marcado por la expansión urbana de Soacha, caracterizada principalmente por la ocupación de espacios rurales para vivienda de estratos bajos, la mayoría de la veces informal y propiciada por la urbanización pirata, que explica la aparición de rentas diferenciales de vivienda, como determinante principal del valor comercial de la tierra.

Las zonas rurales que cuentan con los mayores precios unitarios de la tierra, se encuentran en las inmediaciones del casco urbano y sobre la autopista Sur, con valores que oscilan entre $\$ 100$ y
\$250 millones, asociadas principalmente a la expansión urbana de Soacha y a la presencia de actividades comerciales e industriales, respectivamente.

En Soacha también sobresale la influencia de la carretera que lo comunica con Mondoñedo en el municipio de Bojacá, segmento vial que hace parte del eje que conecta al sur con el norte de la Sabana de Bogotá, marcado con precios cercanos a los \$100 millones. El área rural entre el casco urbano de Soacha y los límites con Bogotá, presenta precios unitarios cercanos a los $\$ 85$ millones, zona que muy probablemente cederá a la expansión urbana de Soacha y Bogotá, para dar paso a la consolidación de un continuo urbano.

\section{Eje T1 Autopista a Medellín}

Este eje se desarrolló con la ampliación y entrega en concesión de la autopista a Medellín, que la convirtió en la principal vía de acceso a Bogotá desde el Magdalena Medio y la Costa Atlántica. Los altos precios unitarios que se presentan a lo largo de este eje, están asociados a la designación de áreas industriales y comerciales en su área de influencia, así como al desarrollo de vivienda para estratos medios y medios - bajos.

Sobresale la zona industrial de Cota, que cubre el sur - occidente del municipio, desde el río Bogotá hasta la carretera Cota - Funza, la cual está dividida por la Autopista a Medellín en dos sectores; el sector sur de la zona industrial llega hasta 
el margen de la laguna de La Florida, en los límites con el municipio de Funza y presenta los más altos precios de la tierra en el eje, con valores que oscilan entre $\$ 300$ y $\$ 700$ millones, montos que también se presentan el área de influencia del Siberia y de la vía que comunica con Tenjo.

Sobre este eje se observa una disminución de los precios de la tierra causada por la presencia del peaje, lo cual se entiende como una respuesta a las restricciones que el pago del uso de la vía, ejerce sobre la circulación diaria de pasajeros y carga, entre los municipios del Occidente y Bogotá.

La aparición de núcleos urbanos sobre la autopista a Medellín en la Vereda La Punta de Tenjo, establece una zona de precios unitarios cercanos a los $\$ 56$ millones. Este tipo de asentamientos urbanos fomenta una especie de metástasis de la ciudad en el primer anillo metropolitano, fenómeno que debe ser controlado a través de la restricción de su crecimiento, mediante el aumento de impuestos prediales y la consolidación de programas de vivienda en los cascos urbanos de los municipio vecinos a Bogotá.

\section{Eje T2 Carretera de Occidente}

Este eje está caracterizado por la presencia de zonas industriales que se establecieron allí desde hace varias décadas. El desarrollo de zonas industriales sobre la autopista de Occidente ha propiciado rentas diferenciales de industria que seguramente determinan los altos precios unitarios de la tierra, aunque notablemente inferiores a los de sobre la vía Funza - Siberia, fenómeno causado por saturación desordenada del espacio entre la carretera y la vía férrea y por la presencia de peajes.

La zona de precios unitarios más elevados del eje, cercanos a $\$ 1.000$ millones, se presenta en el sector ubicado entre la carretera de Occidente y la vía paralela que de Funza conduce al San Andrés Golf Club, hecho que se debe al fenómeno de conurbación que se está dando entre los cascos urbanos de Mosquera y Funza y que explica la generación de rentas diferenciales de vivienda.

La presencia de invasiones en el municipio de Mosquera en los márgenes del río Bogotá, al costado sur del eje, aumenta notablemente los precios de la tierra rural, entre $\$ 85$ y $\$ 170$ millones, a pesar de que este sector cuenta con riesgo de inundación y tiene la influencia del impacto negativo del contaminado río Bogotá.

\section{Precios de la tierra y patrones de uso}

La anterior distribución espacial de los precios de la tierra en los municipios contiguos a Bogotá, permite establecer que éstos están definidos de manera predominante por un patrón de uso del suelo, cuyas principales características son las siguientes: 
- En los municipios del norte, Chía, Sopó, La Calera y Cota, se establecen usos de vivienda para sectores sociales de alto poder adquisitivo con usos complementarios como el de recreación y servicios, los cuales generan rentas de segregación y diferenciales de vivienda, que explican la formación de los más altos precios de la tierra para uso habitacional en la zona de estudio, con valores unitarios que oscilan entre $\$ 250$ y 1.000 millones. Sobresale que la renta diferencial de comercio que entra jugar un papel importante en la formación de los precios de la tierra sobre la Autopista Norte y en la vía que conduce a Cajicá, cuyos valores oscilan entre $\$ 1.500$ y $\$ 2.500$ millones.

- En los municipios del Occidente, Mosquera, Funza y Tenjo, se establecen actividades industriales y de viviendas para el sector intermedio de la sociedad, con usos complementarios, que propician rentas diferenciales de vivienda y de industria y de monopolio de industria. Los usos industriales permiten la formación de precios equiparables a los de las zonas comerciales del norte del área de estudio. La renta de vivienda propicia precios unitarios similares a los del norte, debido a que la mayor fragmentación permite una circulación más rápida del capital.

- Al sur, en el Municipio de Soacha, las principales rentas diferenciales se propician por la presencia de vivienda para sectores bajos de la sociedad.
Los precios de la tierra en el área de estudio, disminuyen a partir de las principales autopistas (autopista Norte, autopista Sur, autopista a Medellín, autopista de Occidente y carretera a La Calera) hasta un valor mínimo, a partir del cual comienzan a subir por efectos de la influencia de alguna de éstas vías o un casco urbano. El caso más sobresaliente se encuentra a ambos costados de la Autopista Norte, como se describe en la figura 2; en este transecto, los mayores precios de la tierra se presentan a ambos costados de la vía y van disminuyendo paulatinamente hasta un mínimo, que en este caso lo determina, al lado oriental las zonas de reserva forestal y al occidental la ronde del río Bogotá; al costado occidental, los precios vuelven a subir por la influencia de las viviendas suburbanas de Calaorra y por la vía que conduce a Cajicá, en donde aparecen rentas diferenciales de vivienda y comercio.

La figura 3 muestra el comportamiento de los precios comerciales de la tierra por influencia de la Autopista a Medellín. Se observa que al costado norte los precios son mayores que al costado sur, como efecto de la designación de la zona para un parque industrial y por la existencia del sector suburbano de Parcelas de Cota. De igual manera, se evidencia la presencia de la laguna de La Florida, afectando los precios de manera fuertemente decreciente. 


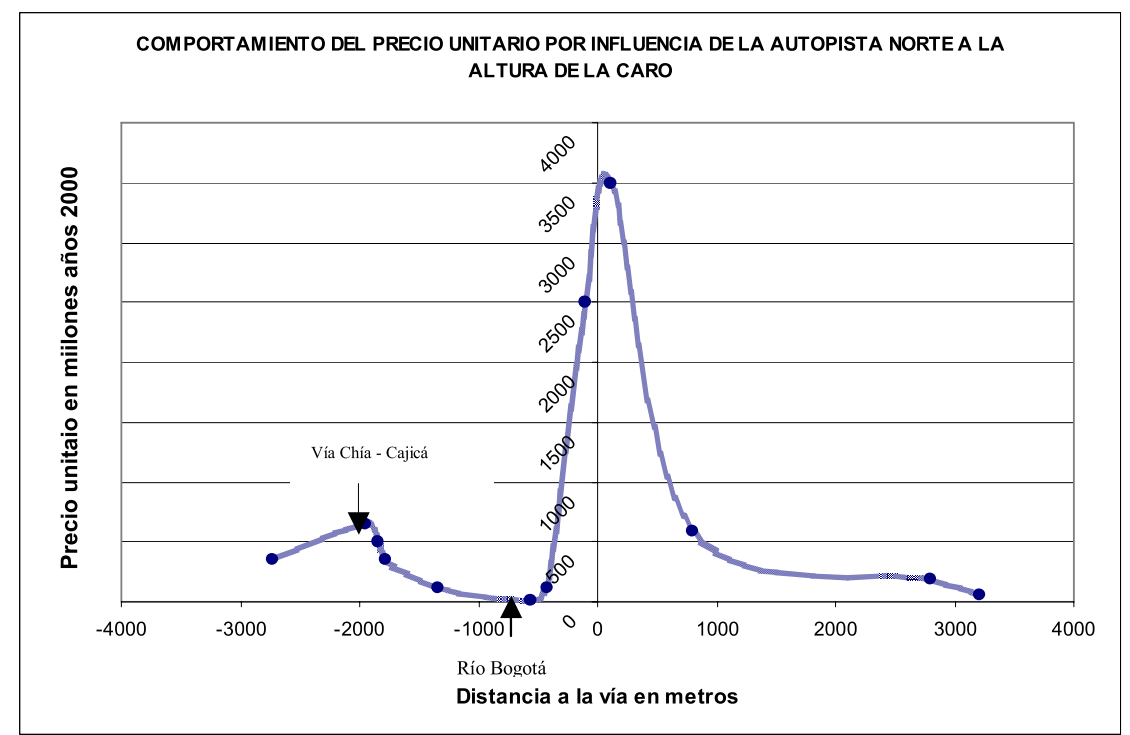

Figura 2. Comportamiento de los precios comerciales unitarios de la tierra por efecto de la Autopista norte. Fuente: datos de campo.

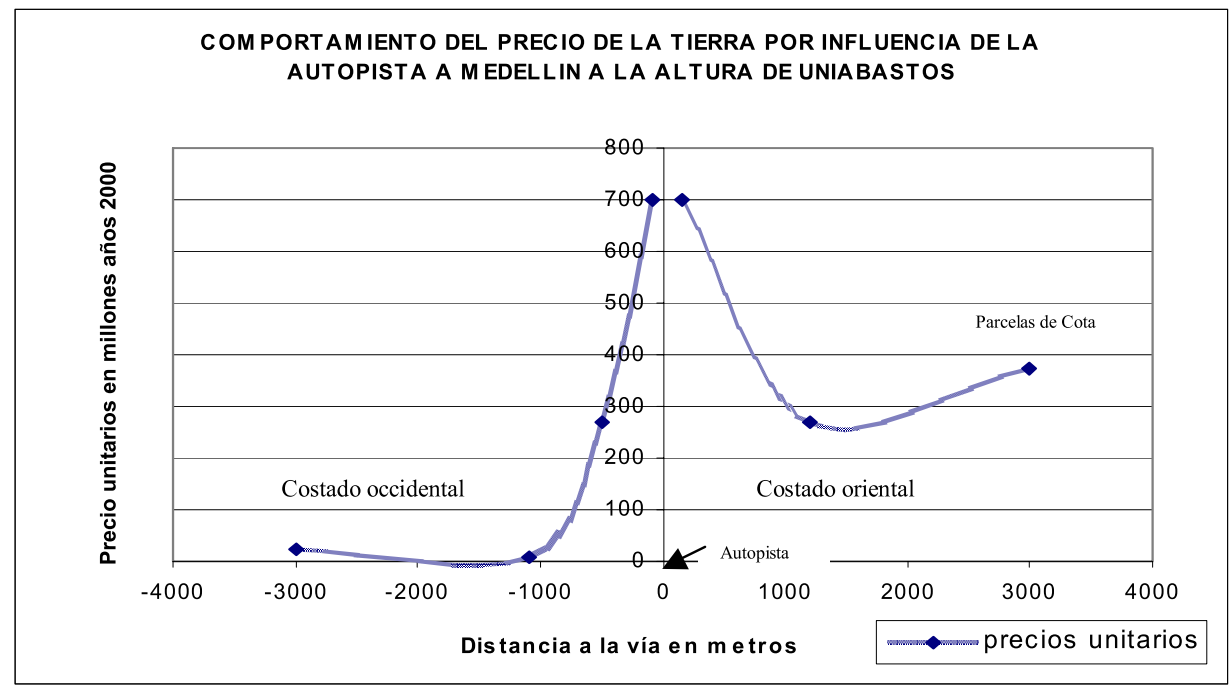

Figura 3. Comportamiento de los precios comerciales unitarios de la tierra por efecto de la Autopista Medellín a la altura de Uniabastos. Fuente: datos de campo.

En la figura 4. se muestra el comportamiento que tienen los precios de la tierra por efecto de la carretera de Occidente y de los cascos urbanos de Mosquera y Funza. Obsérvese que los precios en las inmediaciones de los cascos urbanos son superiores a los que se presentan sobre las vías, lo cual se debe a que las rentas diferenciales de vivienda son muy superiores a las de industria que dominan sobre la vía.

104 Oscar Buitrago Bermúdez 


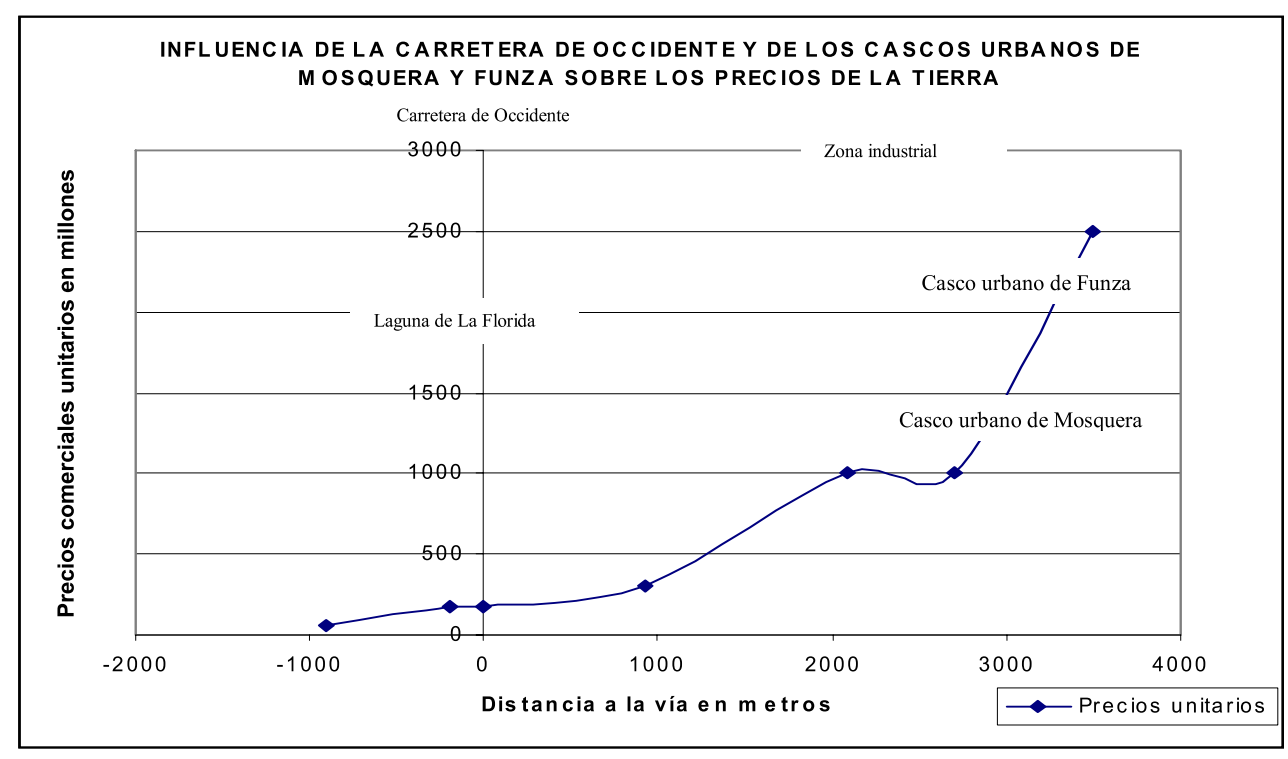

Figura 4. Comportamiento de los precios comerciales unitarios de la tierra por efecto de la carretera de Occidente y de los cascos urbanos de Funza y Mosquera. Fuente: Datos de campo.

\section{Determinacion de los factores formadores del precio de la tierra rural en los municipios contiguos a bogotá}

Se seleccionaron una serie de variables o factores que se esperó tuvieran significancia, en la explicación de la formación del precio de la tierra rural en el área de estudio, los cuales fueron: área del predio, pendiente, presencia de aguas, valor potencial del suelo, forma del predio, calidad de las vías, municipio, distancia a vía pavimentada, distancia a vía más cercana, distancia al casco urbano, distancia al límite político - administrativo de Bogotá, vecino de impacto, uso del suelo y tipo de propietario.
Para la determinar la relación que existe entre el conjunto de variables, se seleccionaron dos técnicas multivariadas complementarias, ellas fueron el análisis de componentes principales y la regresión lineal múltiple. Para la aplicación de los análisis multivariados en primera instancia se definió un universo de estudio constituido por los predios rurales catastrales contenidos en el marco espacial para un total de 20.512 predios catastrales, seguido se diseñó una muestra aleatoria estratificada con afijación óptima para estimar promedios de precio unitarios de la tierra, con un error relativo de $6 \%$ (para el cálculo del tamaño de la muestra, se utilizaron las fórmulas del muestreo aleatorio estratificado de 
Cochran, (1986:144-147), lo cual dio como resultado 995 predios), y por último, se seleccionaron (aleatoriamente) y caracterizaron los predios.

\section{Criterio previo a la aplicación de la técnicas multivariadas}

Para la aplicación de las técnicas de análisis multivariados se tomó como criterio previo la agrupación de predios por municipios, en consideración a que sus estructuras espacial, social y económica los discrimina tanto por su semejanza como por su contigüidad espacial, de la siguiente manera: La Calera-Sopo, Chía, Cota - Tenjo, Funza

- Mosquera y por último, Soacha.

\section{Resultados del análisis de componentes principales}

El empleo del análisis de componentes principales apuntó a interpretar de manera preliminar y síntesis las variables. La aplicación de esta técnica se hizo mediante el procedimiento FACTOR del Sistema de Análisis Estadístico (SAS).

En la Tabla 1 se sintetiza el porcentaje de la contribución de los tres primeros componentes principales de cada subgrupo a la explicación de la variabilidad global de los precios de la tierra. Obsérvese que el análisis de componentes para el subgrupo de Soacha, retiene el máximo porcentaje de explicación, en tanto que el menor porcentaje, lo presenta el subgrupo La Calera - Sopó.

Tabla 1

Contribución de los tres primeros componentes a la variabilidad global

\begin{tabular}{|c|c|c|c|}
\hline GRUPO & COMPONENTE & $\begin{array}{c}\text { \% DE } \\
\text { EXPLICACIÓN }\end{array}$ & $\begin{array}{c}\text { \% TRES } \\
\text { COMPONENTES }\end{array}$ \\
\hline \multirow[t]{3}{*}{ LA CALERA - SOPO } & 1 & 19,4 & \multirow[t]{3}{*}{43,8} \\
\hline & 2 & 13,2 & \\
\hline & 3 & 11,2 & \\
\hline \multirow[t]{3}{*}{ CHIA } & 1 & 25,7 & \multirow[t]{3}{*}{50,1} \\
\hline & 2 & 13 & \\
\hline & 3 & 11,4 & \\
\hline \multirow[t]{3}{*}{ COTA - TENJO } & 1 & 21,6 & \multirow[t]{3}{*}{49,7} \\
\hline & 2 & 15,1 & \\
\hline & 3 & 13 & \\
\hline \multirow[t]{3}{*}{ FUNZA-MOSQUERA } & 1 & 23,3 & \multirow[t]{3}{*}{51,6} \\
\hline & 2 & 16 & \\
\hline & 3 & 12,3 & \\
\hline \multirow[t]{3}{*}{ SOACHA } & 1 & 43,3 & \multirow[t]{3}{*}{72} \\
\hline & 2 & 15,7 & \\
\hline & 3 & 13 & \\
\hline
\end{tabular}

106 Oscar Buitrago Bermúdez 


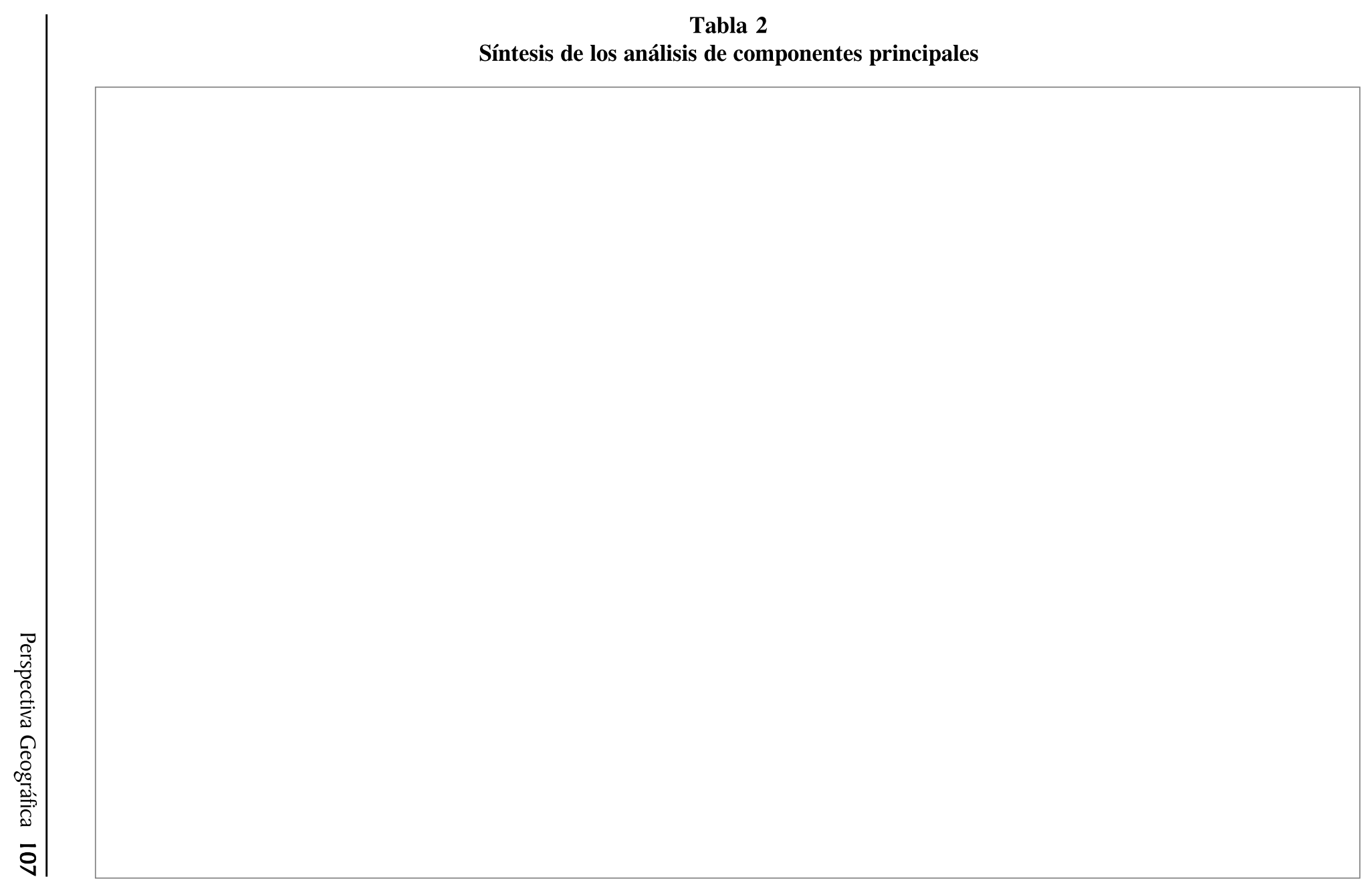


En la Tabla 2, se observa la síntesis que hacen los tres primeros componentes de cada subgrupo. Obsérvese que cada componente sintetiza un conjunto de variables diferente en cada subgrupo de municipios, por lo que se dificulta la identificación de un nombre interpretativo para cada uno de ellos. Por lo anterior, se hace la explicación independiente de los componentes principales retenidos para cada grupo de municipios, buscando sintetizar variables en atributos físicos o artificiales y su relación con la formación del precio de la tierra en cada uno de ellos. De igual manera, se busca retener aquellos atributos comunes a todos los subgrupos de municipios.

\section{Subgrupo La Calera - Sopo}

En este grupo de municipios los dos primeros componentes retienen el $32.6 \%$ de la variación de todas las variables involucradas. Como lo muestra la Tabla 3, el primer componente o de intencionalidad agrícola, está marcando la importancia que tienen los atributos naturales, en tanto que el segundo, definen la intervención de los atributos artificiales o uso del predio.

Tabla 3

Interpretación de los componentes del subgrupo La Calera - Sopó

\begin{tabular}{|l|l|l|}
\hline \multicolumn{1}{|c|}{$\begin{array}{c}\text { NOMBRE } \\
\text { COMPONENTE }\end{array}$} & \multicolumn{1}{|c|}{$\begin{array}{c}\text { ATRIBUTOS DOMINANTES } \\
\text { ASOCIADOS }\end{array}$} & \multicolumn{1}{c|}{$\begin{array}{c}\text { ATRIBUTOS } \\
\text { SECUNDARIOS }\end{array}$} \\
\hline $\begin{array}{l}\text { Componente uno o } \\
\text { de intencionalidad } \\
\text { agrícola. }\end{array}$ & $\begin{array}{l}\text { Físicos relacionados con la calidad agrícola del } \\
\text { terreno: valor potencial del suelo, pendiente y } \\
\text { presencia de agua }\end{array}$ & $\begin{array}{l}\text { Artificiales relacio- } \\
\text { nados con el acceso: } \\
\text { calidad de las vías. }\end{array}$ \\
\hline $\begin{array}{l}\text { Componente dos o de } \\
\text { uso del predio }\end{array}$ & $\begin{array}{l}\text { Artificiales como precio unitario de la tierra, el uso } \\
\text { habitacional y el tipo de propietario jurídico }\end{array}$ & $\begin{array}{l}\text { Uso agropecuario y } \\
\text { tipo de propietario } \\
\text { natural }\end{array}$ \\
\hline
\end{tabular}

Del componente de intencionalidad agrícola del predio se deduce que en estos dos municipios un modelo espacial de precios de la tierra debe asociarse en primer lugar con variables de tipo físico como el valor agrológico, pendiente $\mathrm{y}$ presencia de aguas, lo cual es lógico ya que el uso dominante en estos municipios es el agropecuario; este tipo de atributos aun es importante en el mercado de tierras de la zona.
En cuanto componente uso del predio, al marcarse en él la importancia del uso habitacional y en menor medida el tipo de propietario jurídico, explica la influencia de la creciente entrada a estos municipios de actividades urbanas, dada espacialmente por la presencia de condominios y casas de campo para recreación de fines de semana; en este componente, la actividad agropecuaria marca el

108 Oscar Buitrago Bermúdez 
máximo valor negativo, indicando que este tipo de uso en el subgrupo está asociado a menores precios, en tanto que los mayores precios se asocian al tipo de propietario jurídico y al uso habitacional.

En conclusión, las variables que más variación aportan a los precios de la tierra en estos municipios están relacionadas con atributos físicos del predio para el uso agrícola y en segundo lugar con atributos artificiales como el tipo de propietario y el uso habitacional.

\section{Subgrupo Chía}

En este municipio el primer componente o de intencionalidad agrícola, retiene el $25.7 \%$ de la variabilidad del modelo, el cual asocia, como se observa en la Tabla 4 , los atributos naturales valor potencial del suelo y pendiente con el atributo artificial persona natural, en tanto que en sentido negativo asocia los atributos artificiales accesibilidad y persona jurídica; esto indica que los predios con mayor capacidad de uso agrícola están asociados a personas naturales, en tanto que los predios con mejor acceso están asociados a personas jurídicas. Esta situación es lógica si se considera que la intención de las personas jurídicas se encamina a poseer terrenos bien ubicados que en un futuro puedan ser aprovechados para usos más rentables que la agricultura.

Tabla 4

Interpretación de los componentes del subgrupo Chía

\begin{tabular}{|l|l|l|}
\hline \multicolumn{1}{|c|}{$\begin{array}{c}\text { NOMBRE } \\
\text { COMPONENTE }\end{array}$} & \multicolumn{1}{|c|}{$\begin{array}{c}\text { ATRIBUTOS DOMINANTES } \\
\text { ASOCIADOS }\end{array}$} & \multicolumn{1}{c|}{$\begin{array}{c}\text { ATRIBUTOS } \\
\text { SECUNDARIOS }\end{array}$} \\
\hline $\begin{array}{l}\text { Componente uno o de } \\
\text { inten c i o n a li d a d } \\
\text { agrícola }\end{array}$ & $\begin{array}{l}\text { Intención de uso agropecuarios. Por sus atributos } \\
\text { físicos asociados a la calidad agrícola del terreno y } \\
\text { valor potencial, pendiente y por el artificial, persona } \\
\text { natural. }\end{array}$ & $\begin{array}{l}\text { La accesibilidad con } \\
\text { las variables distancia } \\
\text { a vía pavimenta y a } \\
\text { casco urbano. }\end{array}$ \\
\hline $\begin{array}{l}\text { Componente dos o de } \\
\text { inten c i o n a lid d d d } \\
\text { urbana. }\end{array}$ & $\begin{array}{l}\text { Intención de uso urbano por atributo calidad las vías } \\
\text { y presencia de agua. }\end{array}$ & \\
\hline $\begin{array}{l}\text { Componente tres o } \\
\text { de uso del predio }\end{array}$ & $\begin{array}{l}\text { Artificiales relacionados con el uso actual de la tierra } \\
\text { marcando de manera inversa el habitacional y el } \\
\text { agropecuario. }\end{array}$ & \\
\hline
\end{tabular}


El segundo componente o de intencionalidad urbana, retiene el $13 \%$ de la variabilidad y asocia atributos naturales con artificiales, de la siguiente manera: presencia de aguas con calidad de las vías y propietario jurídico, lo cual indica que este tipo de propietario busca además de una mejor calidad de vías y una buena presencia de aguas.

El tercer componente o de uso del predio, marca una contraposición entre los usos habitacional y agropecuario, indicando que en un modelo de precios de la tierra, estas variables entrarán a actuar inversamente. Es decir que un predio con uso habitacional tendrá mayor precios que un predio con uso agropecuario.

\section{Subgrupo Cota - Tenjo}

El primer componente o de intencionalidad agrícola, de este grupo de municipios, retiene el $21.6 \%$ de la variabilidad, lo cual se relaciona, como se ve en la Tabla 5, con atributos físicos del predio para el uso agropecuario, es decir valor potencial, pendiente y presencia de agua. En este componente, el máximo valor negativo está relacionado con el tamaño del predio, lo cual indica que esta variable juega un papel de carácter inverso en la configuración del modelo de precios de la tierra, situación que se ratifica al mirar el mapa de precios de la tierra del municipio de Cota, que los mayores valores corresponden a predios pequeños.

Tabla 5

Interpretación de los componentes del subgrupo Cota - Tenjo

\begin{tabular}{|l|l|l|}
\hline \multicolumn{1}{|c|}{$\begin{array}{c}\text { NOMBRE } \\
\text { COMPONENTE }\end{array}$} & \multicolumn{1}{|c|}{$\begin{array}{c}\text { ATRIBUTOS DOMINANTES } \\
\text { ASOCIADOS }\end{array}$} & \multicolumn{1}{c|}{$\begin{array}{c}\text { ATRIBUTOS } \\
\text { SECUNDARIOS }\end{array}$} \\
\hline $\begin{array}{l}\text { Componente uno o de } \\
\text { in te n cion a lid a d } \\
\text { agrícola }\end{array}$ & $\begin{array}{l}\text { Naturales relacionados con la calidad agrícola del } \\
\text { terreno: valor potencial del suelo, pendiente, presencia } \\
\text { de vías. }\end{array}$ & $\begin{array}{l}\text { Artificial tamaño del } \\
\text { predio con signo } \\
\text { negativo. }\end{array}$ \\
\hline $\begin{array}{l}\text { Componente dos o } \\
\text { de intencionalidad } \\
\text { urbana. }\end{array}$ & Artificial asociado con la persona jurídica. & \\
\hline
\end{tabular}

110 Oscar Buitrago Bermúdez 
El segundo componente o de intencionalidad urbana, retiene el $15 \%$ de la variabilidad y está asociado a la variable de carácter artificial tipo de propietario. En él la variable persona jurídica de signo positivo, asociada a uso comercial, en contraposición a la variable persona natural de signo negativo, que está vinculada con la variable uso agropecuario, indicando que los predios destinados a actividades agrícolas se encuentran en manos de personas naturales, hecho que muy probablemente incide de manera inversa en el modelo de precio de la tierra, como lo muestra el signo positivo de la variable precio unitario.

\section{Subgrupo Funza - Mosquera}

El primer componente o de intencionalidad urbana con un $23 \%$ de retención de la variabilidad, muestra con signo positivo una alta carga del atributo artificial accesibilidad, dado por las variables distancia al casco urbano y distancia a vía pavimentada; con valores algo menores se presentan las variables uso agropecuario y tipo de propietario natural. Con signo negativo se encuentran con alta variabilidad el tipo de uso actual habitacional y el atributo físico valor potencial, indicando que predios con potencial agropecuario se encuentran con uso habitacional, marcando un uso inadecuado del territorio (ver Tabla 6).

El segundo componente o de uso del predio asocia la variable precio de la tierra con el uso recreacional y en menor proporción, con el tipo de propietario jurídico. Esto se explica por la presencia de importantes clubes en estos dos municipios: San Andrés Golf Club y Club Serrezuela.

Tabla 6

Interpretación de los componentes del subgrupo Funza - Mosquera

\begin{tabular}{|l|l|l|}
\hline \multicolumn{1}{|c|}{$\begin{array}{c}\text { NOMBRE } \\
\text { COMPONENTE }\end{array}$} & \multicolumn{1}{|c|}{$\begin{array}{c}\text { ATRIBUTOS DOMINANTES } \\
\text { ASOCIADOS }\end{array}$} & \multicolumn{1}{c|}{$\begin{array}{c}\text { ATRIBUTOS } \\
\text { SECUNDARIOS }\end{array}$} \\
\hline $\begin{array}{l}\text { Componente uno o } \\
\text { de intencionalidad } \\
\text { urbana }\end{array}$ & $\begin{array}{l}\text { Artificial relacionados con la distancia a casco urbano } \\
\text { y distancia a vía pavimentada. }\end{array}$ & $\begin{array}{l}\text { Artificiales de signo } \\
\text { negativo, relacionadas } \\
\text { con el uso habitacional }\end{array}$ \\
\hline $\begin{array}{l}\text { Componente dos o o } \\
\text { uso del predio }\end{array}$ & $\begin{array}{l}\text { Artificiales relacionadas con el uso recreacional y precio } \\
\text { unitario. }\end{array}$ & \\
\hline
\end{tabular}




\section{Subgrupo Soacha}

El componente uno que retiene 40 $\%$ de la variabilidad, presenta una alta puntuación para el atributo artificial accesibilidad especialmente por la variable distancia al casco urbano, asociada principalmente al uso agropecuario. Los atributos artificiales uso habitacional y forma del predio, marcados con signo negativo, marcan una asociación en contraposición con los de signo positivo (ver Tabla 7).

Tabla 7

Interpretación de los componentes del subgrupo Soacha

\begin{tabular}{|l|l|l|}
\hline \multicolumn{1}{|c|}{$\begin{array}{c}\text { NOMBRE } \\
\text { COMPONENTE }\end{array}$} & \multicolumn{1}{|c|}{$\begin{array}{c}\text { ATRIBUTOS DOMINANTES } \\
\text { ASOCIADOS }\end{array}$} & \multicolumn{1}{c|}{$\begin{array}{c}\text { ATRIBUTOS } \\
\text { SECUNDARIOS }\end{array}$} \\
\hline $\begin{array}{l}\text { Componente uno o de } \\
\text { accesibilidad }\end{array}$ & $\begin{array}{l}\text { Artificiales relacionados con la } \\
\text { accesibilidad y el uso agropecuario }\end{array}$ & $\begin{array}{l}\text { Artificiales de signo negativo } \\
\text { relacionadas con el uso } \\
\text { habitacional y con la forma del } \\
\text { terreno }\end{array}$ \\
\hline $\begin{array}{l}\text { Componente dos o de } \\
\text { propiedad estatal }\end{array}$ & $\begin{array}{l}\text { Artificiales relacionados con el uso } \\
\text { institucional y tipo de propietario } \\
\text { estatal }\end{array}$ & \\
\hline
\end{tabular}

En conclusión, este componente muestra dos puntos extremos en este municipio, uno que indica la presencia de la actividad urbana y otro que expresa el uso rural del territorio. Sobresale la influencia de los predios que están a nombre de entidades estatales y su relación con los precios de la tierra.

\section{Análisis de regresión lineal múltiple de precios de la tierra}

El empleo del análisis de regresión múltiple apunta a identificar, mediante el nivel de dependencia de la variable precios de la tierra que mantiene con las variables independientes, los factores determinantes del precio de la tierra en el área de estudio. La aplicación de esta técnica se hizo mediante el procedimiento STEPWISE del Sistema de Análisis Estadístico (SAS).

Para el uso de esta técnica se consideraron los subgrupos de municipios tenidos en cuenta en el análisis anterior y se estimó importante crear dos grupos más: el correspondiente a la información de los propietarios jurídicos y el conjunto de datos de la muestra. Es decir que se contó con siete bases de datos para el empleo del procedimiento STEPWISE.

112 Oscar Buitrago Bermúdez 
El nivel dependencia que existe entre la variable precio unitario de la tierra y las demás variables seleccionadas, se verificó en las salidas del programa SAS para cada subgrupo, mediante los coeficientes de determinación $\mathrm{R}^{2} \mathrm{o}$ porcentaje de variación de la variable dependiente que es explicado por la regresión $Y_{i}$, los cuales se observan en la Tabla 8. Es necesario anotar que cuando existe un ajuste perfecto entre los valores observados de precio unitario de las tierras y los valores estimados a través de $\mathrm{Y}_{\mathrm{i}}$, el coeficiente es igual a $100 \%$.

Tabla 8

Coeficientes de determinación de las regresiones lineales múltiples

\begin{tabular}{|l|c|}
\hline \multicolumn{1}{|c|}{ SUBGRUPO } & $\begin{array}{c}\text { COEFICIENTE } \\
\text { DE DETERMINACION }\end{array}$ \\
\hline Personas jurídicas de todos los municipios & $86.5 \%$ \\
\hline La Calera - Sopo & $57.0 \%$ \\
\hline Chía & $51.4 \%$ \\
\hline Cota - Tenjo & $69.4 \%$ \\
\hline Funza - Mosquera & $91.0 \%$ \\
\hline Soacha & $99.9 \%$ \\
\hline Toda el área de estudio & $51.4 \%$ \\
\hline
\end{tabular}

De otra parte, de los resultados del procedimiento STEPWISE, se tomaron aquellas variables que más coeficiente de determinación parcial aportan a las diferentes regresiones, las cuales se muestran en la Tabla 9. Es necesario anotar que las regresiones más representativas para el análisis son en su orden las obtenidas de los sugrupos Soacha, Funza - Mosquera, y por último, Personas Jurídicas.

Se observa que en todas las regresiones, las variables que determinan el precio de la tierra son atributos de los predios de carácter artificial, con excepción de la presencia de aguas en el subgrupo Cota - Tenjo y de la pendiente en el Subgrupo Soacha.

Visualizando de manera global, las variables que más coeficiente de determinación aportan a los diferentes modelos, están relacionadas con el atributo artificial accesibilidad, ya sea por la distancia al casco urbano, distancia al límite administrativo con Bogotá, distancia a vía pavimentada o por la calidad de las vías; en segundo lugar, se encuentran las variables relacionadas con el atributo artificial 
tipo de uso, especialmente el recreacional, el agropecuario y el comercial. De manera llamativa, se encuentra la variable municipio de Chía, aportando un alto coeficiente de determinación en el subgrupo personas jurídicas y en la regresión de toda el área de estudio.

En particular para cada subgrupo, el comportamiento es como sigue:

- En el subgrupo Chía los precios de la tierra están relacionados en con variables que determinan la accesibilidad como la distancia al límite de Bogotá, la distancia al casco urbano y la calidad de vías. Variables que explica cerca del $37 \%$ de modelo de regresión lineal múltiple.

- En el subgrupo La Calera Sopó, los precios de la tierra se asocian al uso agropecuario, al uso recreacional y a la distancia al límite de Bogotá. El uso recreacional concuerda con la actual tendencia que se presenta en estos municipios a ser utilizados para el desarrollo de parcelaciones y condominios.

- En el subgrupo Cota Tenjo, las variables que entran jugar papel importante en el precio de la tierra están relacionadas con la accesibilidad y la presencia de aguas, asociado a uso agropecuario, con tendencia a la ampliación del uso habitacional.

- En el subgrupo Funza Mosquera, se presenta el uso recreacional como determinante de los precios de la tierra, causado por la presencia de Clubes, seguido del uso comercial y de la calidad de vías.

- En Soacha, las variables distancia al límite de Bogotá, pendiente y el vecino de impacto entraron a determinar los precios de la tierra. El vecino de impacto se relaciona con la presencia del río Bogotá; la pendiente marca diferencias en los precios debido a que este municipio presenta la mayoría de sus tierras en zonas quebradas, cuyo uso principalmente es agropecuario, en contraposición a las zonas planas afectadas principalmente por la expansión urbana.

En conclusión se puede decir que los principales atributos de los predios que determinan los precios de las tierras en el área de estudio, son de orden artificial y tienen que ver con el tipo de propietario, la accesibilidad y el tipo de uso que tenga el predio. Las figuras 5, 6 y 7 , muestran las curvas de los valores estimados de precios de la tierra a través de estos modelos de regresión lineal múltiple $\mathrm{Y}_{\mathrm{i}}$ y los valores de precios de la tierra observados. 


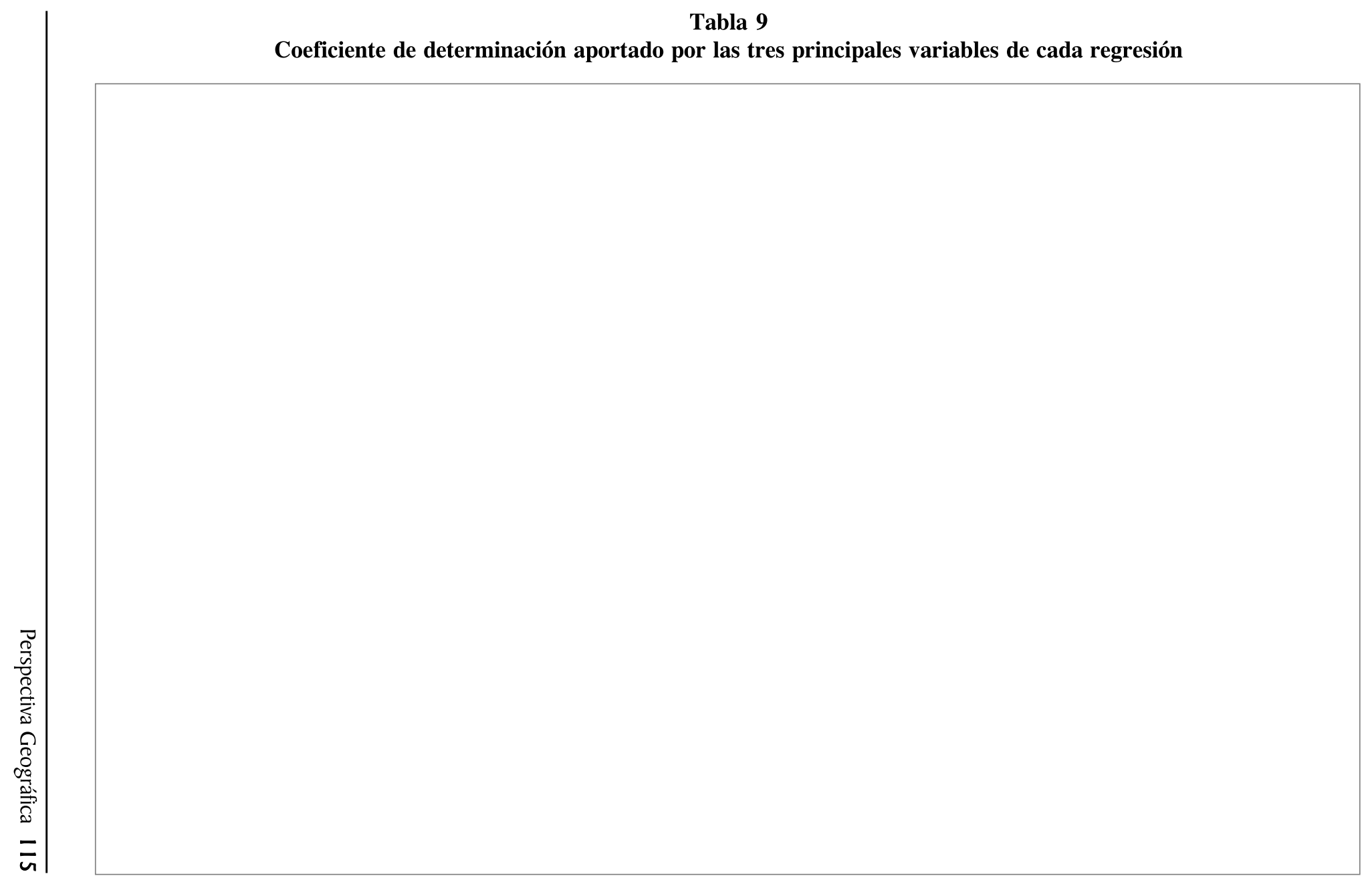




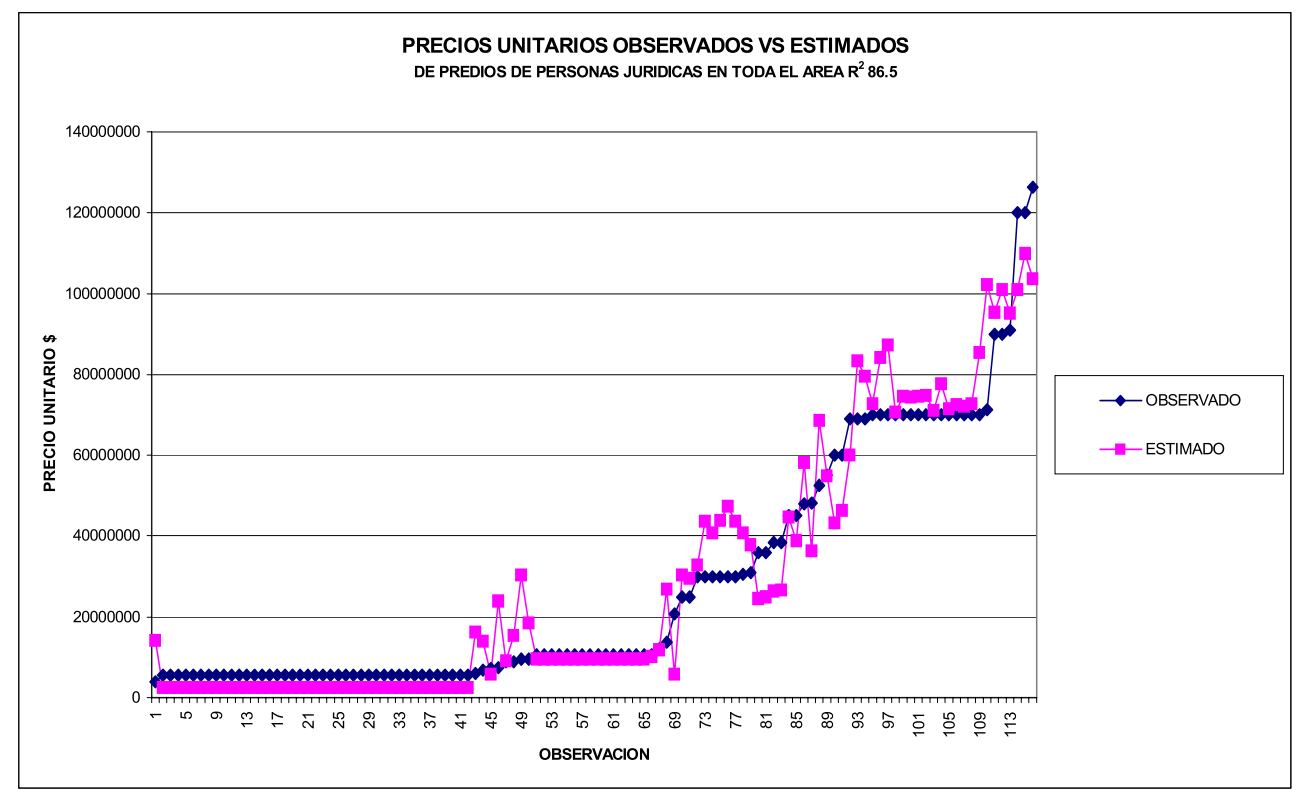

Figura 5. Curvas de la regresión lineal múltiple subgrupo SOACHA y de datos observados.

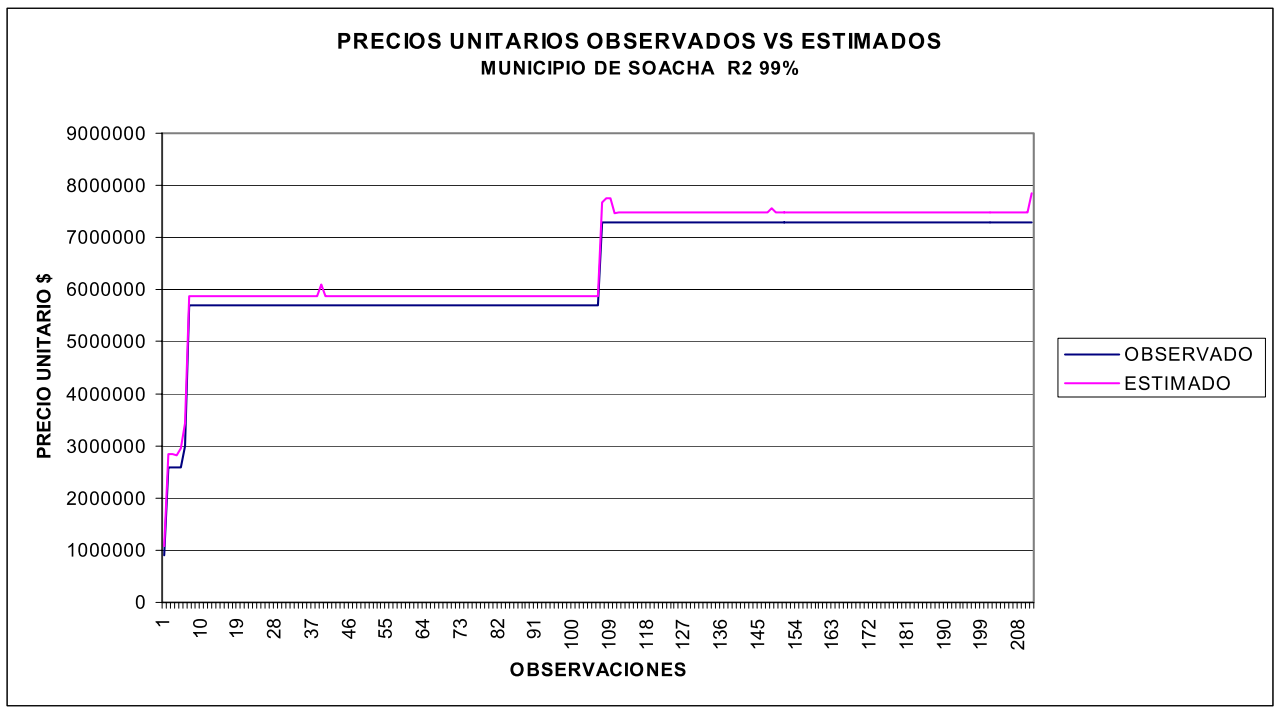

Figura 6. Curvas de la regresión lineal múltiple del subgrupo PERSONAS JURÍDICAS y de datos observados.

116 Oscar Buitrago Bermúdez 


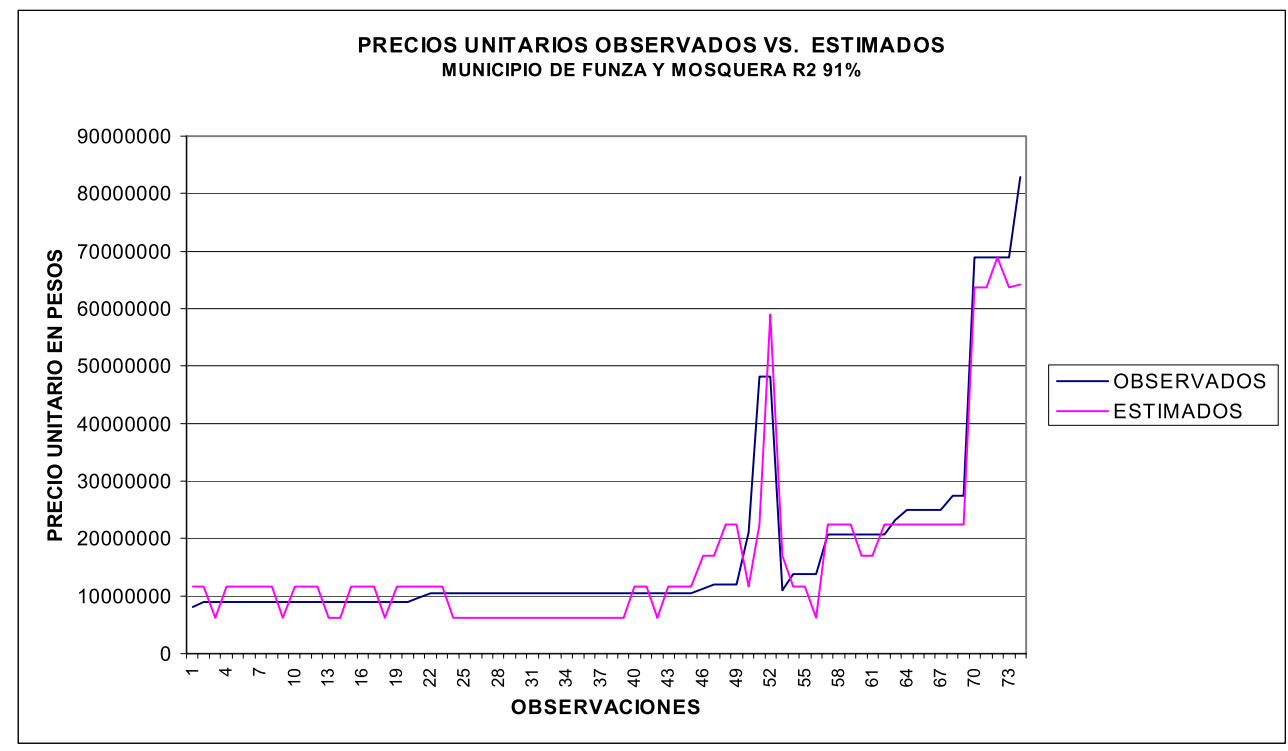

Figura 7. Curvas de la regresión lineal múltiple del subgrupo FUNZA - MOSQUERA y de datos observados.

\section{Conclusiones}

Del análisis de componentes se deduce que en los municipios contiguos a Bogotá se presenta una agrupación de variables determinada principalmente por el tipo de propietario. El hecho de que el tipo de propietario jurídico se asocie con mayor frecuencia al uso habitacional de los predios rurales en los municipios contiguos a Bogotá, hace pensar en que existe una clara intención de ellos para continuar con su actuación urbanizadora, sin importar el estrato social que consumirá el espacio construido. Lo anterior se ratifica si se observa que en casi todos lo municipios, los propietarios jurídicos guardan una considerable reserva de tierras, expresada en cerca del $38 \%$ del área ocupada por predios mayores de 20 hectáreas. Como se observa en la Tabla 10, Soacha presenta el mayor porcentaje, La Calera y Tenjo los menores, en tanto que los demás municipios poseen valores muy similares, que oscilan entre 13 y $14 \%$. 
Tabla 10

Área en manos de personas jurídicas y en predios de más 20 hectáreas

\begin{tabular}{|l|c|}
\hline MUNICIPIO & $\begin{array}{c}\text { \% DELAREA EN } \\
\text { PERSONAS JURIDICAS } \\
\text { ( > 20Ha) }\end{array}$ \\
\hline CHIA & 14,3 \\
\hline COTA & 14,3 \\
\hline FUNZA & 13,1 \\
\hline LA CALERA & 3,3 \\
\hline MOSQUERA & 16,5 \\
\hline SOACHA & 24,1 \\
\hline SOPO & 13,4 \\
\hline TENJO & 0,9 \\
\hline
\end{tabular}

Fuente: cálculos del autor a partir de datos catastro del IGAC, 1998.
En términos generales los análisis multivariados de los atributos de los predios seleccionados, permiten deducir que las características físicas del terreno, tales como calidad del suelo, pendiente y presencia de aguas no son el mayor factor en la conformación del precio de la tierra rural en el área de estudio, sino la expectativa de urbanización, que se evidencia a través de atributos artificiales relacionados con la accesibilidad, el tipo de uso actual y el tipo de propietario, los cuales orientan el mercado de tierras hacia la satisfacción de las necesidades de ocupación del espacio por parte de los agentes urbanizadores o personas jurídicas.

118 Oscar Buitrago Bermúdez 


\section{Bibligrafía}

AVILA BERNAL, Alvaro. Elementos de análisis de la función regional. Universidad de los Andes. Instituto de Estudios Sociales. Programa de planificación y docencia en planificación del desarrollo regional. Bogotá, 1978.

BARRERÈ, P. Urbanización del campo en los países industrializados. En: BARRERÈ, P. et all. Espacios rurales y urbanos en áreas industrializadas. Barcelona, 1988. Editorial Oikos-tau, S.A - II Congreso Mundial Vasco.

BORRERO, Oscar y DURAN Esperanza. El valor del suelo urbano y sus implicaciones en el desarrollo de la ciudad: análisis del caso de Bogotá. CENAC. Bogotá 1980.

BORRERO, Oscar y DURAN Esperanza. El valor del suelo urbano en Bogotá 1959 - 1988 y sus implicaciones en el desarrollo de la ciudad. FEDELONJAS. Bogotá, 1988.

BORRERO, Oscar y DURAN Esperanza. El valor del suelo urbano en Bogotá 1988 1996. Análisis de la evolución de los precios desde 1960. LONJA DE PROPIEDAD RAIZ DE BOGOTA. Santa Fe de Bogotá, 19996.

BUSTAMENTE L., Francisco D. Manual de la contribución de valorización. Editorial Teoría del Color. Medellín, 1996.

CETRO DE ESTUDIOS ECONÓMICOS y CAMARA DE COMERCIO DE BOGOTÁ. Bogotá - Sabana. Un territorio posible. CCC - Universidad de Los Andes. Bogotá, 1998.

COCHRAN, William. Técnicas de muestreo. Editorial CECSA. México, D.F. 1986.

ESTEBANEZ A., J. y BRADSHAW P., R. Técnicas de cuantificación en geografía. Editorial Tebar Flores. Madrid, 1978.

DEPARTAMENTO ADMINISTRATIVO NACIONAL DE ESTADÍSTICAS. Censo 1993. 
GARZA, Gustavo. Modos de producción y urbanización. En : KINGMAN G., Eduardo. Las ciudades en la transición al capitalismo. Universidad Autónoma de México. México D.F., 1988.

GILBERT, Alan \& GUGLER, Josef. Cities poverty and development urbanization in the world.2da edition. New York. Oxfor University Press.1992.331o.

GOBERNACION DE CUNDINAMARCA. Anuario estadístico 1999. Comité departamental de estadísticas. Bogotá, 2000.

INSTITUTO GEOGRAFICO AGUSTIN CODAZZI. Subdirección de Catastro Nacional. Metodología de zonas homogéneas. Primera parte. Bogotá, 1987.

INSTITUTO GEOGRAFICO AGUSTIN CODAZZI. Subdirección de Catastro Nacional. Metodología para la definición y avalúo de zonas homogéneas (área urbana 1985) Documento de discusión. División de Investigación e instrucción catastral. Bogotá, 1985.

INSTITUTO GEOGRAFICO AGUSTIN CODAZZI. Subdirección de Catastro Nacional. Proyecto de mejoramiento del proceso de conservación catastral. Información catastral sistematizada. Instrumento para transcripción de datos. Bogotá, 1998.

JARAMILLO, Samuel. Hacia una teoría de la renta del suelo urbano. Ediciones UniandesInstituto Geográfico Agustín Codazzi. Santafé de Bogotá, 1994.

JOHNSON, James. Geografía urbana. Segunda edición. Barcelona, 1980.

LORENTE, Luis. COLOMBIA. MINISTERIO DE AGRICULTURA. OFICINA DE PLANEAMIENTO DEL SECTOR AGROPECUARIO. Urbanización y producción agropecuaria: documento para discusión. Bogotá Ministerio de Agricultura.1976. p. 67.

MENDEZ Ricardo. Geografía Económica. La lógica espacial del capitalismo global. Ariel Geográfica. Barcelona, 1997.

MISION SIGLO XXI. El futuro de la capital. Un estudio de prospectiva urbana. Alcaldía Mayor de Santa Fe de Bogotá , D.C.

MOHAM Rakesh y VILLAMIZAR Rodrigo. Evolución de los precios de la tierra en el contexto de un rápido crecimiento urbano. En : PACHON MUÑOZ Alvaro. Lecturas sobre economía urbana. Bogotá, 1986. Fundación Simón Bolívar - Instituto de Estudios Políticos.

MONTANEZ G., Gustavo. Hacia donde va la Sabana. Editorial SENA CES Universidad NacionaL. Bogotá, 1994.

120 Oscar Buitrago Bermúdez 
PUJADAS R. y FONT J. Ordenación y planificación territorial. Colección espacios y sociedades. Editorial Síntesis. Madrid, 1998.

RAMOS G., Wilson. Predial, valorización y plusvalía en el distrito capital. Nuevo tributo denominado "plusvalía" y la contribución por beneficio local. Editorial LEGIS. Santafé de Bogotá, 1999.

RODRIGUEZ C, María Luisa. El valor del suelo y competencia por la ocupación espacial. Area de influencia de la Autopista Norte (Calle 200 - Briceño). Tesis de grado para optar al título de Mágister en Geografía con énfasis en ordenamiento territorial. EPG, Convenio UPTC-IGAC. Bogotá, 1999.

SAMPER S., Arturo. Banco de Tierras. Aplicaciones en Colombia. Departamento Nacional de Planeación. Documento de trabajo. Bogotá, 1994.

SAMPER GNECCO, Patricio. Expansión urbana y vivienda. En CAMACOL. Vol 13. No. 3. Edición No. 44. Septiembre de 1990.

UNIVERSIDAD NACIONAL DE COLOMBIA. FACULTAD DE ARTES. Seminario de ecología y urbanización. Ecología y expansión urbana. Ponencia presentada por la Universidad Nacional. Bogotá, 1973.

VALENZUELA RUBIO, Manuel. Urbanización y crisis rural en la sierra de Madrid. Instituto de estudios en Administración Local. Madrid, 1977.

VIEILLE Paul. Teherán. Mercado del suelo y sociedad urbana. Editorial Gustavo Gill. Barcelona, 1974.

VINUESA A. J. y DOMINGUEZ V., M. J. Los procesos de Urbanización. Editorial Sintesis.Colección Espacios y Sociedades. Madrid, 1991.

ZARATE M., Antonio. El espacio interior de la ciudad. Editorial Síntesis. Colección Espacios y Sociedades. Madrid, 1991. 
